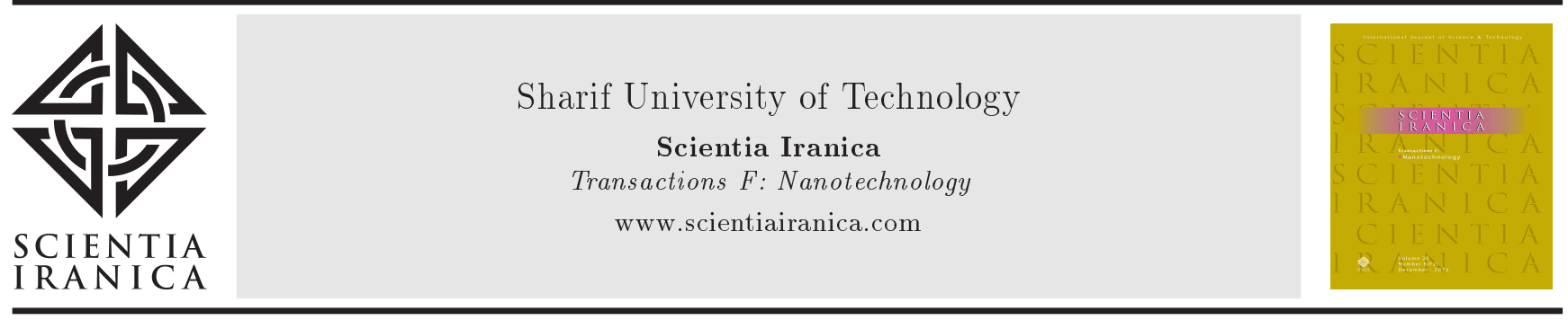

\title{
Relaxation time assessment in nanoparticle containing bimodal and mono-disperse shear thickening fluids
}

\author{
M. Fahool and A.R. Sabet* \\ Department of Composites, Iran Polymer and Petrochemical Institute (IPPI), Tehran, P.O. Box 14965/115, Iran.
}

Received 12 July 2014; received in revised form 9 September 2015; accepted 16 January 2016

\section{KEYWORDS}

Shear thickening

fluids;

Mono-disperse;

Bimodal;

Nano;

Relaxation time.

\begin{abstract}
When an applied shear to a fluid results in steep rise in its viscosity, it is referred to as Shear Thickening Fluid (STF). In this study, we investigate rheological response of bimodal against mono-dispersed nano sized silica particles in an STF system. Two sizes of nano-silica particles (50 nm fumed silica and $12 \mathrm{~nm}$ colloidal silica) were dispersed in polyethylene glycol in various compositions and were compared with monodispersed STF. Results showed lower critical shear rate for shear thickening in bimodal nanoparticle containing STF than mono-dispersed STF. Higher viscosities were also observed in different compositions of bimodal system. Examination of both STF systems under oscillatory stress condition revealed faster response by the bimodal system. Results also indicated that the storage and loss modulus for the bimodal STF system had low critical angular frequency compared to mono-dispersed STF. Study of relaxation time spectrum diagram for the two STFs showed that relaxation time for mono-dispersed STF was faster than that for bimodal STF system.

(C) 2016 Sharif University of Technology. All rights reserved.
\end{abstract}

\section{Introduction}

Shear-Thickening Fluids (STFs) are concentrated colloidal suspensions composed of non-aggregating solid particles suspended in fluids. These suspensions have the property of increasing fluid viscosity with increasing shear rate [1].

STFs have been the focus of attention for the scientists involved in processing technology, and not many consider that they offer positive advantages in industrial situations.

Initially, interest in shear thickening derived mainly from its association with damage to processing equipment, e.g. particle aggregation, which is known to compromise a range of properties. However, due to its highly nonlinear behaviour, the potential for damping application and machine mounts was explored $[2,3]$. In the past two decades, extensive research effort has

*. Corresponding author. Tel.: 021 48662130

E-mail address: a.sabet@ippi.ac.ir (A.R. Sabet) been directed towards understanding the rheological behaviour of STF, including study of different particles' size, geometry, concentration, and continuous phase viscosity. Keller and Keller [4] investigated a bimodal mixture of coal particles at 0.77 and $7.9 \mu \mathrm{m}$ and observed an increase in volumetric particle concentration compared to mono-disperse suspension, but no shear thickening tendencies were reported for the bimodal suspension. Wagstaff and Chaffey [5] showed that the suspensions of larger particles thickened at lower shear rates and polydispersity increased the critical shear rate and reversible thickening only in systems that had predominately repulsive interactions. Experimental evidence by small angle neutron scattering and light scattering by Bender and Wagner [6] showed that shear thickening occurred without the shear induced order-disorder transition. They also found out that measurements on bimodal mixtures verified the scaling laws derived from the force balance and provided a strategy for controlling the shear thickening transition. Raghavan and Khan [7] investigated shear thicken- 
Table 1. Physical properties of nano-particles used in this study.

\begin{tabular}{ccccc}
\hline Nano-particle & $\begin{array}{c}\text { Particle size } \\
(\mathbf{n m})\end{array}$ & $\begin{array}{c}\text { Bulk density } \\
(\mathbf{g} / \mathbf{l})\end{array}$ & $\begin{array}{c}\text { BET } \\
\left(\mathbf{m}^{2} / \mathbf{g}\right)\end{array}$ & Chemical name \\
\hline Silica OX50 & 40 & 70 & $50 \pm 15$ & $\begin{array}{c}\text { Hydrophilic fumed } \\
\text { silica } \mathrm{SiO}_{2}\end{array}$ \\
Silica 406 & 12 & 50 & $200 \pm 25$ & Colloidal silica $\mathrm{SiO}_{2}$ \\
\hline
\end{tabular}

ing as well as strain thickening behaviour of nonflocculated fumed silica suspensions in polypropylene glycol under oscillatory shear. They reported two distinct regimes of strain thickening with first occurring at high critical strains and low frequencies and the second occurring at high critical frequencies and a constant lower strain. Maranzano and Wagner [8] predicted scattering SANS spectra for polydispersed hard sphere, which conformed to the hard sphere interactions in experimental results. Maranzano and Wagner [9] affirmed that the hydrocluster formation resulted in the shear thickening transition. Brown et al. [10] demonstrated that shear thickening could be masked by a yield stress and could be recovered when the yield stress decreased below a threshold. During the period 2002-2010, most research on shear thickening fluids has been on the usage of STFs in some applications, in particular damping and impact application. Waitukaitis and Heinrich [11] in their study published in the journal of Nature demonstrated that these stresses originated from an impact-generated solidification front that transformed an initially compressible particle matrix into a rapidly growing jammed region, ultimately leading to extraordinary amounts of momentum absorption. They assert that "prior interpretations of the impact resistance as dominated by shear thickening need to be revisited". Crawford et al. [12] exhibited irreversible thickening behaviour by investigating fumed silica slurries; it appears to be a complex interplay between cation hydration, silica surface hydration, and hydrodynamic shear forces. Fahool and sabet [13] observed the shear induced transient hydrocluster formation in shear thickening fluids using UV-visible spectrum. Their study showed that the amount of aggregations in bimodal STF was higher than that in the mono-disperse mode. They attributed this to the interaction of more particles, i.e. the presence of smaller particles among the larger particles. Lee et al. [14] showed rheology and microstructure of concentrated hard sphere suspensions in the liquid and crystal coexistence region by the lattice Boltzmann method coupled with the smoothed profile method. Their result clearly showed how strong the ordered structure was in relation to the shear stress distortion.

Despite extensive research report about STF behaviour, little attention has been given to bimodality effect on STF behaviour in a particular relaxation time after shear stress removal. No report in open literature was found on the assessment of relaxation time and reversibility rate for the STF systems. This study examine bimodal combination of nano sized silica particles in a polyethylene glycol as a shear thickening fluid. The particular focus of the work is on relaxation time associated with the fluid containing bimodal silica particles and their comparison with monodispersed system.

\section{Materials and method}

In order to study the response of shear thickening fluid containing bimodal particles, two types of nanoparticle sized hydrophilic silica were used for the preparation of the Shear Thickening Fluid (STF). Fumed silica OX50 from Evonic-Degussa (Germany) and colloidal silica 406 from West system (UK) were used as suspending agents. Table 1 provides details for the nano-particles used. Polyethylene glycol (PEG) with molecular weight of $400 \mathrm{~g} / \mathrm{mol}$ from Sanaye Shimiyaee Kimiyagaran Emrooz (Iran) was used as the continuous phase for preparing the STF. Neat polyethylene glycol has a viscosity of $120 \mathrm{mPa} . \mathrm{s}$ and density of $1.126 \mathrm{~g} / \mathrm{ml}$ at $20^{\circ} \mathrm{C}$. The reason for selecting $\mathrm{PEG}$ is that it is a non-toxic, easy to handle, and thermally stable material and its flow behavior is of Newtonian nature.

\subsection{STF preparation}

As all the received nano-silica particles are in agglomerated state, careful steps must be taken to ensure uniform dispersion of the particles in the continuous phase; this task becomes even more complicated when two particles of different sizes are to be added together. Predetermined amounts of nano-size silica OX50 and colloidal silica 406 were consecutively mixed with $50 \mathrm{ml}$ of PEG using a mechanical mixer at 1000-100 rpm (depending on nanoparticle concentration) for a period of 2 hrs; the compound was then irradiated with highintensity ultrasonic horn (Ti-horn, $20 \mathrm{kHz}, 90 \mathrm{~W}$, $30 \mathrm{~mm}$ ) for $10 \mathrm{~min}$. The samples were then placed in a vacuum oven for $24 \mathrm{hrs}$ to eliminate the air bubbles generated during mixing operation. Mono-disperse STF compounds containing 35, 40, and 45 wt\%. OX 50 silica were also prepared in the same manner for comparison. Weight ratios for bimodal system $35 \mathrm{wt} \%$ are $20 \mathrm{wt} \%$ fumed silica OX50 (20FS) and $15 \mathrm{wt} \%$ Colloidal silica 406 (15CS). 


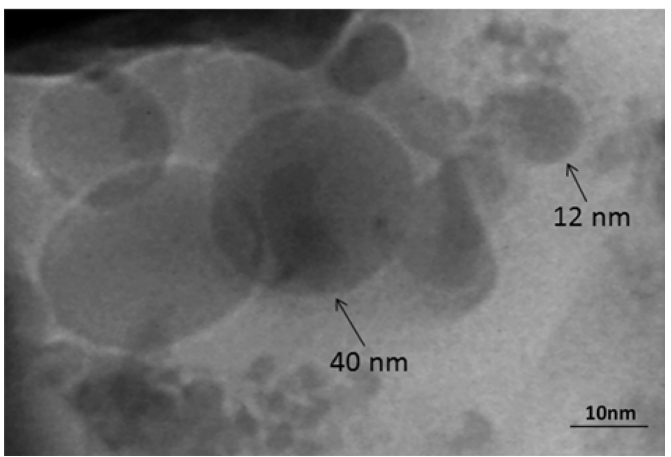

Figure 1. TEM micrograph of nano-particle in 40 and $12 \mathrm{~nm}$.

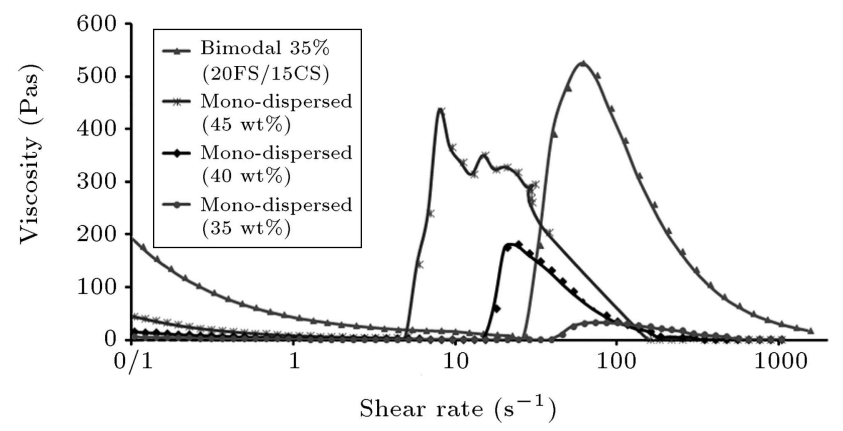

Figure 2. Rheological behavior of STFs with total particle compositions of 35,40 , and $45 \mathrm{wt} \%$ for mono-dispersed and $35 \mathrm{wt} \%$ for bimodal systems.

\subsection{Characterization}

The prepared STF samples were investigated for rheological properties using rheometer MCR-501. Tests were conducted at room temperature in a steady state flow mode and shear rate ramp of $0.01-1000 \mathrm{~s}^{-1} \mathrm{using}$ a cone plate of size $50 \mathrm{~mm}$ and angle of $1^{\circ}$. Minimum of three tests has been run on each specimen and the average value has been reported, here. The silica particles distribution was investigated using transition electron microscopy. Figure 1 shows TEM micrograph of the nano-particle used in this study.

\section{Results and discussion}

Figure 2 shows rheological behavior of polyethylene glycol fluid containing nano-size bimodal and monodispersed silica particle comprising 406 colloidal silica and OX50 fumed silica with total particle compositions of 35, 40, and $45 \mathrm{wt} \%$ for mono-dispersed and $35 \mathrm{wt} \%$ for bimodal system. This shear thickening characteristic occurs when dispersed solid particles in the continuous medium are in contact and hydrocluster phenomenon forms this result in transient fluctuations of particles. This transient fluctuation of particles results in frictions between particles and leads to abrupt increase in fluid viscosity.

The figure presents three distinct regions; a relative shear thinning happens at low shear rate for all systems containing silica compositions and at high shear rates, the fluid experiences a sudden transition with an increase in viscosity, which signifies shear thickening behavior and finally a shear-thinning-like behavior. The critical shear rate at which shear thickening occurs in both compositions is 1 to $100 \mathrm{~s}^{-1}$. A similar line of research on shear thickening fluid for other materials has also been reported. Franks et al. [15] demonstrated that critical shear rate of silica and alumina suspensions containing 60 wt\% were 72 and $600 \mathrm{~s}^{-1}$, respectively. Also, Brown and Jaeger [16] reported that the critical shear rate of cornstarch in $60 \mathrm{wt} \%$ was almost $0.1 \mathrm{~s}^{-1}$. Therefore, comparing the effective shear rate domains and critical shear rates in different mixtures included in the current research reveals considerable variability. This difference in behavior between the shear thickening fluids may be attributed to the differences in surface energy and surface groups on the particles.

Generally, STF materials have good reversibility characteristic after shear stress removal, but controlling the rate of reversibility allows wider application of the STF. This reversibility is directly related to the speed of steady state transition after shear stress removal, i.e. returning from hydrocluster condition; this is vital when energy absorption applications are envisaged for these types of materials. This reversibility can be investigated by considering relaxation time spectrum diagram, where relaxation time $(\tau)$ versus $H(\tau) \tau$ is considered. Here, $H(\tau)$ is the relaxation time spectrum that is associated with the dynamic parameters such as the storage and loss modulus. The relaxation time spectrum is a quantity characteristic describing the viscoelastic properties of polymer melts and polymer solutions [17]. For determination of this function, i.e. $(H(\tau))$, various empirical formulations have been proposed [18,19], e.g. Alfrey approximation [20]. He presented an approximation for estimation of $H(\tau)$; see Eq. (1):

$$
G(t)=\int_{0}^{\infty} \frac{H(\tau)}{\tau} e^{-t / \tau} d \tau=\int_{-\infty}^{+\infty} H(\tau) e^{-t / \tau} d \ln \tau
$$

where $G(t)$ is stress relaxation modulus at the time $t$ (modulus obtained from a stress-relaxation experiment, where the sample is held at constant strain and the decaying stress is recorded). This parameter is related to the storage and loss moduli that are calculated by Eqs. (2) and (3):

$$
\begin{aligned}
& G^{\prime}(\omega)=\int_{0}^{\infty} H(\tau) \frac{\omega^{2} \tau^{2}}{1+\omega^{2} \tau^{2}} \frac{d \tau}{\tau} \\
& G^{\prime \prime}(\omega)=\int_{0}^{\infty} H(\tau) \frac{\omega \tau}{1+\omega^{2} \tau^{2}} \frac{d \tau}{\tau} .
\end{aligned}
$$

And so $G(t)$ is computed in the following way: 


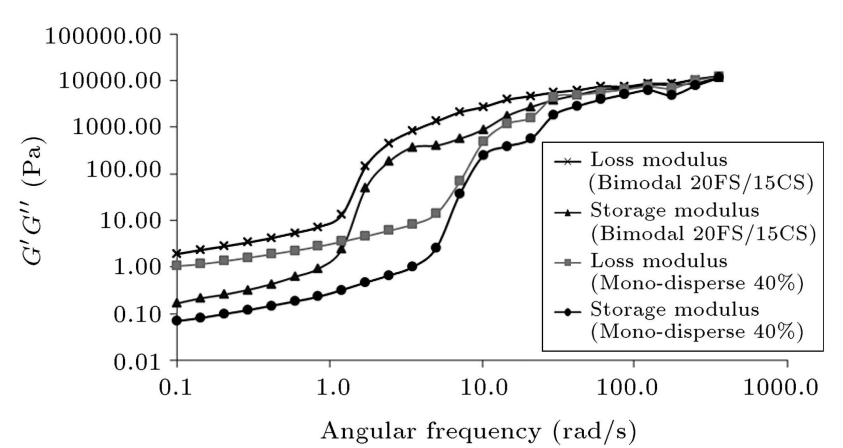

Figure 3. Storage $\left(G^{\prime}\right)$ and loss $\left(G^{\prime \prime}\right)$ moduli versus angular frequencies for the two STF systems.

$$
\begin{aligned}
G(t) & \simeq G^{\prime}(\omega)-\sum a_{k} G^{\prime \prime}\left(2^{k} \omega\right)-\sum b_{k}\left\{G^{\prime}\left(2^{k+1} \omega\right)\right. \\
& \left.-G^{\prime}\left(2^{k} \omega\right)\right\}
\end{aligned}
$$

where $\omega$ is angular frequency, $a_{k}$ and $b_{k}$ are numerical constants that are to be chosen in such a way that the formulae become as accurate as possible [21].

Figure 3 depicts storage $\left(G^{\prime}\right)$ and loss $\left(G^{\prime \prime}\right)$ moduli behavior for the two STF systems in different angular frequencies.

Figure 3 shows that exactly at the point where complex viscosity experiences sudden increase, the storage and loss modulus also increases. Further study of the figure indicates that the storage and loss modulus for the bimodal STF system has a lower critical angular frequency than that of mono-dispersed system.

Estimation of the relaxation time spectrum $H(\tau)$ from Eq. (1) is only possible by the Laplace transform or the Fourier transforms formulation. In this work, the rheometer's software directly calculates $H(\tau)$ while performing frequency sweep test. The peak point in the diagram indicates relaxation time for the concerned material. The sharpness in the curve's peak indicates close relaxation times for the particles, i.e. the whole STF material relaxes at the same time. On the other hand, not very sharp peak shows non-uniform relaxation times by the material. Relaxation times for both STF materials are presented in Figures 4 and 5 . Comparison of relaxation times for both STF materials reveals that the relaxation time of mono-dispersed $\operatorname{STF}(\tau=0.089 \mathrm{~s})$ is faster than that of bimodal $\operatorname{STF}(\tau=0.33 \mathrm{~s})$. This may be directly attributed to closer packing of particles in bimodal system, i.e. small diameter particles being located between larger particles. This closer packing in bimodal STF results in higher friction between particles, which in turn leads to longer time for relaxation and return to the original state condition.

To give better physical meaning to our argument, an epoxy resin was also used to compare relaxation time spectrum between them; see Figure 6 .

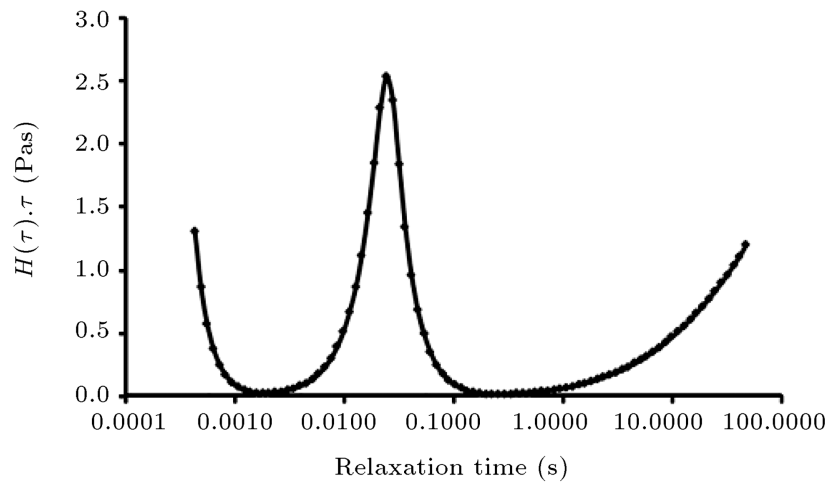

Figure 4. Relaxation time for mono-disperse system at $40 \mathrm{wt} \%$.

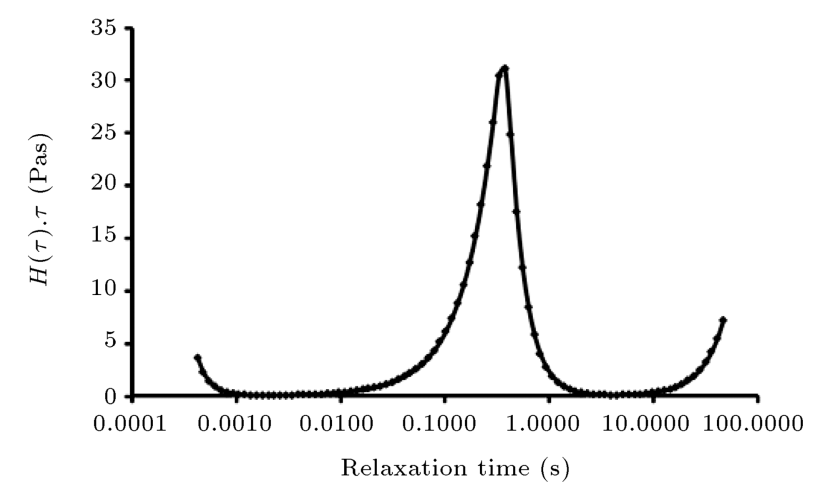

Figure 5. Relaxation time for bimodal system at $35 \mathrm{wt} \%$.

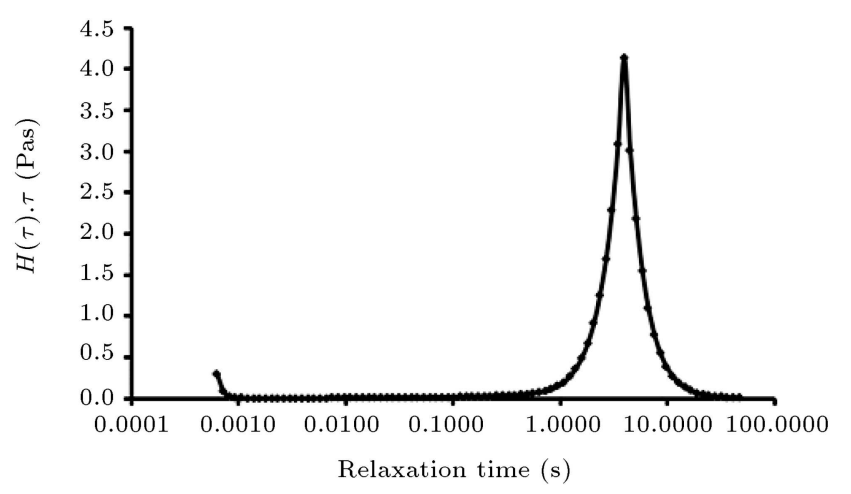

Figure 6. Relaxation time curve for epoxy resin.

In Table 2, the data related to relaxation times of the shear thickening fluid in different modes are given for comparison. The table shows reversibility time of epoxy resin at $\tau=3.93 \mathrm{~s}$ compared to that of $\tau=0.089$ $\mathrm{s}$ for the mono-dispersed STF and that of $\tau=0.33 \mathrm{~s}$ for the bimodal particle containing STF. Comparison of the three $\tau$ values indicates that relaxation time speed of shear thickening fluids is more than that of epoxy resin, while the initial viscosities of STF and epoxy resin are approximately equal. Therefore, relaxation time of STFs is much less than that of conventional fluids. These results are consistent with the observed shear thickening behaviour.

Table 2 shows that based on the trend of change 
Table 2. Relaxation time for different fluids.

\begin{tabular}{cccccc}
\hline $\begin{array}{c}\text { Epoxy 828 } \\
\text { (pure) }\end{array}$ & $\begin{array}{c}\text { Bimodal STF } \\
\mathbf{3 5} \text { wt\% }\end{array}$ & $\begin{array}{c}\text { Mono-dispersed } \\
\text { STF 45 wt\% }\end{array}$ & $\begin{array}{c}\text { Mono-dispersed } \\
\text { STF 40 wt\% }\end{array}$ & $\begin{array}{c}\text { Mono-dispersed } \\
\text { STF 35 wt\% }\end{array}$ & $\begin{array}{c}\text { Sample } \\
\text { type }\end{array}$ \\
\hline 3.93 & 0.33 & 0.0164 & 0.089 & 0.22 & Relaxation time(s) \\
\hline
\end{tabular}

in relaxation time for a mono-disperse fluid, reduction in the relaxation time associated with an increased concentration of the dispersed phase is probably due to the increase in shear thickening property caused by increasing dispersed particle concentration. Also, comparison of the relaxation time $(\tau)$ values indicates that the relaxation time speed of shear thickening fluids is more than that of epoxy resin, while the initial viscosities of STF and epoxy resin are approximately equal.

Therefore, relaxation time of STFs is much less than that of conventional fluids. These results are consistent with the observed shear thickening behavior.

\section{Conclusions}

Rheological behavior of bimodal shear thickening fluid made of two sizes of nanoparticles and mono-dispersed system was successfully investigated. Hydrocluster formation, onset of shear thickening, critical shear stress, elastic and damping response, reversibility, and relaxation time spectrum are among the considered parameters. The result shows better interaction between particles in the bimodal system, thus, demonstrating the strong shear thickening transfer at a shear rate lower than that of mono-dispersed system and also at lower concentration. Comparison of bimodal combinations with mono-disperse systems shows superior properties of the bimodal STFs in terms of increase in viscosity for all the corresponding composition ratios tested.

Oscillatory stress condition revealed faster response by the bimodal system. The storage and loss modulus of the bimodal STF system has a lower critical angular frequency than that of mono-dispersed STF. Comparison between relaxation times of both STF materials reveals that mono-dispersed STF has less relaxation time than bimodal STF. Relaxation time is one of the main factors in damping applications. With this study, we are able to design STF damping system with specific relaxation time for use in sensitive applications.

\section{Nomenclature}

$\begin{array}{ll}\tau & \text { Relaxation time } \\ H(\tau) & \text { Relaxation time spectrum } \\ t & \text { Time } \\ G(t) & \text { Stress relaxation modulus }\end{array}$

$\begin{array}{ll}\omega & \text { Angular frequency } \\ G^{\prime} & \text { Storage modulus } \\ G^{\prime \prime} & \text { Loss modulus }\end{array}$

$G^{\prime \prime} \quad$ Loss modulus

\section{References}

1. Barnes, H. "Shear-thickening ('Dilatancy') in suspensions of nonaggregating solid particles dispersed in Newtonian liquids", J. Rheol, 33(2), pp. 329-366 (1989).

2. Helber, R., Doncker, F. and Bung, R. "Vibration attenuation by passive stiffness switching mounts", Journal of Sound and Vibration, 138(1), pp. 47-57 (1990).

3. Laun, H.M., Bung, R., Hess, S., Loose, W., Hess, O., Hahn, K. and Lindner, P. "Rheological and small angle neutron scattering investigation of shear-induced particle structures of concentrated polymer dispersions submitted to plane Poiseuille and Couette flowa", $J$. Rheol, 36(4), pp. 743-787 (1992).

4. Keller, D.S. and Keller, D.V. "The effect of particle size distribution on the antithixotropic and shear thickening properties of coal-water dispersions", Journal of Rheology, 35(8), p. 1583 (1991).

5. Wagstaff, I. and Chaffey, C.E. "Shear thinning and thickening rheology: I. Concentrated acrylic dispersions", Journal of Colloid and Interface Science, 59(1), pp. 53-62 (1977).

6. Bender, J. and Wagner, N.J. "Reversible shear thickening in monodisperse and bidisperse colloidal dispersions", Journal of Rheology, 40(5), p. 899 (1996).

7. Raghavan, S.R. and Khan, S.A. "Shear-thickening response of fumed silica suspensions under steady and oscillatory shear", Journal of Colloid and Interface Science, 185(1), pp. 57-67 (1997).

8. Maranzano, B.J. and Wagner, N.J. "The effects of interparticle interactions and particle size on reversible shear thickening: Hard-sphere colloidal dispersions", Journal of Rheology, 45(5), p. 1205 (2001).

9. Maranzano, B.J. and Wagner, N.J. "Flow-small angle neutron scattering measurements of colloidal dispersion microstructure evolution through the shear thickening transition", The Journal of Chemical Physics, $\mathbf{1 1 7}(22)$, p. 10291 (2002).

10. Brown, E., Forman, N.A., Orellana, C.S., Zhang, H., Maynor, B.W., Betts, D.E. and Jaeger, H.M. "Generality of shear thickening in dense suspensions", Nature Materials, 9(3), pp. 220-4 (2010).

11. Waitukaitis, S.R. and Jaeger, H.M. "Impact-activated solidification of dense suspensions via dynamic jamming fronts", Nature, 487(7406), pp. 205-9 (2012). 
12. Crawford, N. et al. "Shear thickening and shearinduced agglomeration of chemical mechanical polishing slurries using electrolytes", Rheologica Acta, 52(5), pp. 499-513 (2013).

13. Fahool, M. and Sabet, A.R. "UV-Visible assessment of hydrocluster formation and rheological behaviour in bimodal and mono-disperse shear thickening fluids", Rheologica Acta, 54(1), pp. 77-83 (2015).

14. Lee, Y.K., Nam, J., Hyun, K., Ahn, K.H. and Lee, S.J. "Rheology and microstructure of non-Brownian suspensions in the liquid and crystal coexistence region: Strain stiffening in large amplitude oscillatory shear", Soft Matter, 11(20), pp. 4061-74 (2015).

15. Brown, E. and Jaeger, H.M. "A dynamic jamming point for shear thickening suspensions", Physical Review Letters, 103, p. 4 (2009).

16. Franks, G., Zhou, Z., Duin, N.J. and Boger, D.V. "Effect of interparticle forces on shear thickening of oxide suspensions", Journal of Rheology, 44(4), pp. 759-79 (2000).

17. Roths, T., Maier, D., Friedrich, C., Marth, M. and Honerkamp, J. "Determination of the relaxation time spectrum from dynamic moduli using an edge preserving regularization method", Rheologica Acta, 39(2), pp. 163-173 (2000).

18. Bird, R.B., Armstrong, R.C. and Hassager, O. "Dynamics of polymeric liquids", Fluid Mechanics, 1 (1987).

19. Chhabra, R.P. and Richardson, J.F. "Non-Newtonian flow and applied rheology: Engineering applications", Butterworth-Heinemann (2008).
20. Ward, I.M. and Sweeney, J., An Introduction to the Mechanical Properties of Solid Polymers, 4th Ed., pp. 73-75, John Wiley \& Sons, Ltd, England (2005).

21. Schwarzl, F.R. "Numerical calculation of stress relaxation modulus from dynamic data for linear viscoelastic materials", Rheologica Acta, 14(7), pp. 581-590 (1975).

\section{Biographies}

Masoud Fahool is an MSc degree candidate in the Department of Composites at Iran Polymer and Petrochemical Institute, Tehran, Iran. He received his BSc degree in Chemical Engineering in 2013 from Arak University. His research interests include composite structure and processing, rheological behavior of polymers, and high velocity impact on polymer composites.

Ali Reza Sabet, PhD, is Associate Professor in Composite Structure in the Department of Composites at Iran Polymer and Petrochemical Institute, affiliated to the Ministry of Science, Research, and Technology. He received a BSc degree in Mechanical Engineering and MSc degree in Solid Mechanics from Manchester University Institute of Science and Technology, Manchester, UK, in 1988 and 1990, respectively, and a PhD degree in Polymer Engineering from Iran Polymer and Petrochemical Institute, Tehran, Iran, in 2008. His research interests include composite structure, rheological behavior of polymer, high velocity impact on polymer composites, and light structures. 